\title{
On some majorization of derivatives in the class $S^{*}(\gamma)$
}

\author{
by ANDRZEJ WRZESIEŃ (Łódź)
}

Abstract. We give a generalization of some result of J. Janowski and J. Stankiewicz $[2]$.

Introduction. Let $S^{*}(\gamma)(|\gamma|<\pi / 2)$ denote the class of $\gamma$-spiral-starlike functions, i.e., functions $F(z)$ holomorphic in the disc $K_{1}=\{z ;|z|<1\}$ and satisfying the conditions

$$
F(0)=0, \quad F^{\prime}(0)=1, \quad \operatorname{Re}\left\{e^{-i \gamma} \frac{z F^{\prime}(z)}{F(z)}\right\}>0, \quad z \in K_{1} .
$$

Among the many results concerning this class we have the sharp estimation [1]

$$
m_{1}(r, \gamma) \leq\left|\frac{z F^{\prime}(z)}{F(z)}\right| \leq m_{2}(r, \gamma)
$$

where

$$
\begin{gathered}
m_{1}(r, \gamma)=\frac{\sqrt{1+2 r^{2} \cos 2 \gamma+r^{4}}-2 r \cos \gamma}{1-r^{2}} \\
m_{2}(r, \gamma)=\frac{\sqrt{1+2 r^{2} \cos 2 \gamma+r^{4}}+2 r \cos \gamma}{1-r^{2}}, \\
r=|z|<1, \quad F \in S^{*}(\gamma) .
\end{gathered}
$$

Let $H$ denote the class of holomorphic functions in $K_{1}$. Define $\Omega=$ $\left\{w \in H ;|w(x)| \leq 1\right.$ for $\left.z \in K_{1}\right\}$.

Let $f_{1}, f_{2}$ be two holomorphic functions in $K_{1}$. We say that $f_{1}$ is $m a$ jorized by $f_{2}$ in $K_{R}=\{z ;|z|<R\}$ and write $f_{1} \ll f_{2}$ if there exists a holomorphic function $\phi$ such that $|\phi(z)| \leq 1$ and $f_{1}(z)=\phi(z) f_{2}(z), z \in K_{R}$.

J. Janowski and J. Stankiewicz [2] considered the following problem. Let $A, B$ be two fixed classes of holomorphic functions in $K_{1}$. Find the smallest

1991 Mathematics Subject Classification: Primary 30C45. 
function $T(r)=T(r, A, B), r \in[0,1)$, such that for every pair of functions $f \in A, F \in B$ we have the implication

$$
f \ll F \text { in } K_{1} \Rightarrow\left|f^{\prime}(z)\right| \leq T(r, A, B)\left|F^{\prime}(z)\right| \text { for }|z|=r<1 .
$$

Main result. Let $f \in H$ and $F \in S^{*}(\gamma)$.

TheOREM. If $f \ll F$ in $K_{1}$ and $|z|=r<1$, then

(4) $\left|f^{\prime}(z)\right| \leq T\left(r, H, S^{*}(\gamma)\right)\left|F^{\prime}(z)\right|$, where

(5) $T\left(r, H, S^{*}(\gamma)\right)=\left\{\begin{array}{r}\text { for } r \in\left[0, r^{*}\right], \\ \frac{r^{4}+8 r^{2} \cos ^{2} \gamma+2 r^{2}-4 r P(r, \gamma) \cos \gamma+1}{4 r(P(r, \gamma)-2 r \cos \gamma)} \\ \text { for } r \in\left(r^{*}, 1\right),\end{array}\right.$

(6) $r^{*}=\sqrt{3+4 \cos \gamma-2 \sqrt{2+6 \cos \gamma+4 \cos ^{2} \gamma}}$,

(7) $P(r, \gamma)=\sqrt{1+2 r^{2} \cos 2 \gamma+r^{4}}$.

The result is sharp.

Proof. If $f \ll F$ in $K_{1}$ then there exists $\phi \in \Omega$ such that

$$
f(z)=\phi(z) F(z) \quad \text { for } z \in K_{1} .
$$

Differentiating (8) and dividing by $F^{\prime}(z)$ we obtain

$$
\frac{f^{\prime}(z)}{F^{\prime}(z)}=\phi^{\prime}(z) \frac{F(z)}{F^{\prime}(z)}+\phi(z) .
$$

Applying the well-known estimation for $\phi \in \Omega$,

$$
\left|\phi^{\prime}(z)\right| \leq \frac{1-|\phi(z)|^{2}}{1-|z|^{2}} \quad \text { for } z \in K_{1}
$$

we have from (9), (1) and (10)

$$
\left|\frac{f^{\prime}(z)}{F^{\prime}(z)}\right| \leq \frac{1-|\phi(z)|^{2}}{1-r^{2}} \cdot \frac{r\left(1-r^{2}\right)}{\sqrt{1+2 r^{2} \cos 2 \gamma+r^{4}}-2 r \cos \gamma}+|\phi(z)| .
$$

Define $u=|\phi(z)|$ and denote the right hand side of the above inequality by $G(u)$. Thus

$$
G(u)=-\frac{r}{P(r, \gamma)-2 r \cos \gamma} u^{2}+u+\frac{r}{P(r, \gamma)-2 r \cos \gamma}
$$

where $P(r, \gamma)=\sqrt{1+2 r^{2} \cos 2 \gamma+r^{4}}$.

The function $G(u)$ attains its maximum at $u=1$ when $r \in\left[0, r^{*}\right]$ and at

where

$$
u=\frac{P(r, \gamma)-2 r \cos \gamma}{2 r} \quad \text { when } r \in\left(r^{*}, 1\right)
$$

$$
r^{*}=\sqrt{3+4 \cos \gamma-2 \sqrt{2+6 \cos \gamma+4 \cos ^{2} \gamma}} .
$$


Therefore

$$
\max _{u \in[0,1]} G(u)=\left\{\begin{array}{r}
\begin{array}{l}
\text { for } r \in\left[0, r^{*}\right] \\
r^{4}+8 r^{2} \cos ^{2} \gamma+2 r^{2}-4 r P(r, \gamma) \cos \gamma+1
\end{array} \\
4 r(P(r, \gamma)-2 r \cos \gamma) \\
\text { for } r \in\left(r^{*}, 1\right)
\end{array}\right.
$$

Thus by (12) and (11) we have (4).

The result is sharp. For $r \in\left(0, r^{*}\right]$ and for every pair of functions $\left\{f(z)=e^{i \theta} F(z), F(z)\right\}, F \in S^{*}(\gamma), \theta$ an arbitrary real number, we have $T\left(r, H, S^{*}(\gamma)\right)=1$.

If $r \in\left(r^{*}, 1\right)$ equality is achieved at $z_{0}=r e^{i \theta_{0}}$ for the functions $\widehat{f}(z)=$ $\widehat{\phi}(z) \widehat{F}(z)$ and $\widehat{F}(z)$, where

$$
\begin{gathered}
\widehat{\phi}(z)=\frac{z+\alpha}{1+\alpha z}, \quad \alpha=\frac{-2 r^{2}-2 r \cos \gamma+P(r, \gamma)}{r(2+2 r \cos \gamma-P(r, \gamma))}, \\
\widehat{F}(z)=\frac{z}{\left(1+z e^{-i \theta_{0}}\right)^{2 e^{i \gamma} \cos \gamma}}, \quad \widehat{F} \in S^{*}(\gamma) .
\end{gathered}
$$

By simple calculation we can check that

$$
\left|\widehat{f}^{\prime}\left(z_{0}\right) / \widehat{F}^{\prime}\left(z_{0}\right)\right|=T\left(r, H, S^{*}(\gamma)\right)
$$

and this completes the proof.

Re mark. For $\gamma=0$ we obtain a result of [2] (Theorem 2, p. 54).

\section{References}

[1] C. Bucka and K. Ciozda, Some estimations and problems of the majorization in the classes of functions $S_{(\alpha, \beta)}^{k}$, Ann. Univ. Mariae Curie-Skłodowska 29 (4) (1975), $29-41$.

[2] J. Janowski and J. Stankiewicz, A relative growth of modulus of derivatives for majorized functions, ibid. 32 (4) (1978), 51-61.

INSTITUTE OF MATHEMATICS

TECHNICAL UNIVERSITY OF ŁÓDŹ

AL. POLITECHNIKI 11

90-924 ŁÓDŹ, POLAND 